\title{
DESENVOLVIMENTO E AVALIAÇÃO DA ESTABILIDADE FÍSICO-QUÍMICA DE UM XAMPU CONTENDO EXTRATOS DE EUTERPE OLERACEA MART. E BERTHOLLETIA EXCELSA H.B.K.
}

\section{DEVELOPMENT AND EVALUATION OF PHYSICAL-CHEMICAL STABILITY OF A XAMPU CONTAINING EXTRACTS OF EUTERPE OLERACEA MART. AND BERTHOLLETIA EXCELSA H.B.K.}

\author{
G. T. P. SILVA ${ }^{1}$ e G. F. da SILVA ${ }^{1,2}$ \\ ${ }^{1}$ Universidade do Estado do Amazonas - UEA, Escola Superior de Tecnologia \\ ${ }^{2}$ Universidade do Federal do Amazonas - UFAM, Departamento de Química, Programa de \\ Pós-Graduação em Química \\ E-mail: jeffacanha@gmail.com
}

\author{
article info \\ Article history: \\ Received 20 May 2016 \\ Accepted 3 January 2017 \\ Available online 27 July 2017
}

PALAVRAS-CHAVE:Xampu Fotoprotetor; Açaí; Planejamento Estatístico.

KEYWORDS: Photoprotector shampoo; Açai; Statistical P.

\begin{abstract}
RESUMO: Os filtros solares têm como objetivo prevenir doenças geradas pelo excesso de sol, reduzindo a quantidade de radiação ultravioleta absorvida pela pele e também couro cabeludo. A indústria cosmética tem investido na produção de produtos de origem natural, incluindo os xampus. Um planejamento estatístico deve ser empregado quando o produto desenvolvido possui mais de uma variável e precisa-se obter a melhor resposta nos testes realizados. Desta forma, no presente trabalho, o qual analisou as propriedades fisicoquímicas e organolépticas do xampu fotoprotetor com extratos de açai e castanha-do-pará, fez-se o uso de um planejamento fatorial fracionário com ponto central para verificar quais das variáveis (lauril éter sulfato de sódio, dietanolamina de ácido graxo de coco e anfótero betaínico) influenciam nas características do xampu desenvolvido. No teste de centrifugação, algumas formulações apresentaram precipitado. O lauril éter sulfato de sódio foi o componente que mais influenciou resposta de condutividade elétrica. A partir dos resultados, conclui-se que o planejamento experimental foi eficaz para triar os componentes que afetam nas características das formulações.
\end{abstract}

\begin{abstract}
Sunscreens are meant to prevent diseases caused by excess sun, reducing the amount of ultraviolet radiation absorbed by the skin and also the scalp. The cosmetic industry has invested in the production of products of natural origin, including shampoos. Statistical planning should be employed when the developed product has more than one variable and it is necessary to obtain the best response in the tests performed. Thus, in the present work, which analyzed the physico-chemical and organoleptic properties of the photoprotective shampoo with extracts of açai and Brazil nuts, we used a fractional factorial design with a central point to verify which of the variables Sodium lauryl ether sulfate, coconut fatty acid diethanolamine and amphoteric betaine) influence the characteristics of the developed shampoo. In the centrifugation test, some formulations presented precipitate. Sodium lauryl ether sulfate was the component that most influenced electrical conductivity response. From the results, it is concluded that the experimental design was effective to screen the components that affect the characteristics of the formulations.
\end{abstract}




\section{INTRODUÇÃO}

Típico fruto da região amazônica, o açaí (Euterpe Oleracea Mart.) possui diversos benefícios a saúde, proveniente das suas funções fitoquímicas e da sua atividade antioxidante (Portinho et al., 2012). O extrato de açaí também apresenta uma excelente ação fotoprotetora, a qual reduz os efeitos causados pela radiação emitida pela luz solar quando entra em contato com a pele e o cabelo. (Soares et al., 2015; Giokas et al., 2005).

O extrato da castanha-do-pará (Bertholletia excelsa), também típica da região Amazônica, possui ação emoliente, nutritiva e lubrificante, sendo assim aplicada na indústria cosmética e dermacêutica (Pastore et al, 2005).

O xampu é um produto cosmético extremamente útil e muito procurado pelos consumidores. Seu principal objetivo é efetuar a limpeza ou fixação de substâncias no couro cabeludo e também nos fios do cabelo, tendo como função retirar da superfície capilar impurezas provenientes de secreções, resíduos celulares e do meio externo. Xampus que possuem, além da função de limpeza, uma ação farmacológica são chamados de xampus de tratamento. (Fujiwara et al., 2009).

Faz-se o uso de um planejamento experimental quando se deseja otimizar um processo que tenha duas ou mais variáveis, através de uma análise de todas as possíveis interações entre as variáveis existentes a fim de se obter um ponto ótimo (Rocha, 2010).

Dessa forma, o objetivo do presente trabalho foi desenvolver e analisar a estabilidade físico-química de formulações de xampu que contêm extratos de açaí e aastanha-do-pará através de um planejamento fatorial com ponto central.

\section{METODOLOGIA}

\subsection{Planejamento Experimental}

A fim de otimizar o processo de desenvolvimento e formulação do xampu fotoprotetor utilizou-se de um planejamento fatorial completo com ponto central, verificando os efeitos gerados pelos três fatores em três diferentes níveis de resposta, simbolizados por (-1) para o nível inferior, $(+1)$ para o nível superior e $(0)$ para o ponto central. Realizaram-se oito ensaios (23) em duplicata e um ponto central em triplicata.

As respostas analisadas foram condutividade e características organolépticas. $\mathrm{Na}$ Tabela 1 encontram-se os dados quantitativos e qualitativos das formulações elaboradas. 
Tabela 1- Quantidades (em gramas) dos fatores utilizados em cada formulação e seus respectivos níveis.

\begin{tabular}{cccc}
\hline Formulação & $\begin{array}{c}\text { Lauril éter sulfato } \\
\text { de sódio }\end{array}$ & $\begin{array}{c}\text { Dietanolamina de } \\
\text { ácido graxo de coco }\end{array}$ & Anfótero betaínico \\
\hline 1 & $27(-1)$ & $3(-1)$ & $6(+1)$ \\
2 & $42(+1)$ & $6(+1)$ & $3(-1)$ \\
3 & $27(-1)$ & $6(+1)$ & $6(+1)$ \\
4 & $42(+1)$ & $6(+1)$ & $6(+1)$ \\
5 & $42(+1)$ & $3(-1)$ & $3(-1)$ \\
6 & $27(-1)$ & $3(-1)$ & $3(-1)$ \\
7 & $27(-1)$ & $6(+1)$ & $3(-1)$ \\
8 & $42(+1)$ & $3(-1)$ & $6(+1)$ \\
9 & $34,5(0)$ & $4,5(0)$ & $4,5(0)$ \\
10 & $34,5(0)$ & $4,5(0)$ & $4,5(0)$ \\
11 & $34,5(0)$ & $4,5(0)$ & $4,5(0)$ \\
\hline
\end{tabular}

\subsection{Desenvolvimento das Formulações}

As formulações desenvolvidas foram baseadas em formulações já preparadas no trabalho de Silva et al. (2014).

Em um béquer, os componentes da formulação foram adicionados e agitados manualmente com o auxílio de uma espátula, seguindo a ordem descrita: inicialmente fez-se a diluição do laurel éter sulfato de sódio na água em banho-maria à $80^{\circ} \mathrm{C}$, logo após foram acrescentados o anfótero e a dietanolamina; em seguida adicionou-se a formulação os conservantes, o EDTA (quelante), a ureia e o mentol previamente triturado. Por fim foram acrescentou-se o surfax, os extratos de açaí e castanha-do-pará e a essência de açaí.

\subsection{Análises Físico-Químicas}

Condutividade elétrica: Estudou-se a condutividade elétrica $(\mathrm{k} \Omega / \mathrm{cm})$ através de um condutivímetro digital CG 1800 da marca Gehaka. O eletrodo foi inserido em uma dispersão aquosa a $10 \%(\mathrm{p} / \mathrm{p})$ da amostra em água destilada. O teste foi realizado em triplicada, realizando a limpeza do eletrodo com água destilada a cada amostra analisada (Casteli et al., 2008).

Centrifugação: Utilizou-se uma centrífuga Centrifuge 5702 da marca Eppendorf, que gera um estresse na amostra, fazendo uma simulação do aumento na força gravitacional, aumentando, consequentemente, a mobilidade das partículas e prevendo possíveis instabilidades (Faria et al., 2012).

Foram depositados $10,00 \mathrm{~g}$ da amostra em tubos de ensaios tampados, específicos para o teste de centrifugação, que foram submetidos a três ciclos de centrifugação com diferentes velocidades de rotação (1000 rpm, $2500 \mathrm{rpm}, 3500 \mathrm{rpm})$ com duração de 30 minutos cada ciclo. $\mathrm{O}$ teste foi realizado em duplicata.

Após este teste, analisou-se as características organolépticas e possíveis alterações 
físicas, como formação de precipitado (Faria et al., 2012; Casteli et al., 2008).

\subsection{Avaliação de Xampus Comerciais}

Para se criar uma base de dados comparativos para verificar a qualidade das formulações desenvolvidas, foram submetidos, os xampus comerciais das marcas Haskell Cosméticos Naturais; Alfaparf Milano; Johnson's Baby; Nazca; Seda; Suissa e Natura SPA, aos mesmos testes físico-químicos.

\subsection{Análise Estatística dos Resultados}

Para a análise estatística dos dados experimentais foi utilizado o Software Statistica 10. Utilizou-se a Análise de Variância (ANOVA) para avaliação da concordância das médias dos resultados do planejamento experimental. O intuito desta análise é observar se existe alguma diferença muito significativa entre as médias e também se os fatores influenciam em alguma variável independente. Para descobrir os fatores que influenciam significativamente na resposta estudada fez-se o gráfico de Pareto (Lins, 1993).

\section{RESULTADOS E DISCUSSÃO}

Os resultados obtidos nos testes de centrifugação, condutividade elétrica e o aspecto organoléptico das formulações desenvolvidas estão expostos na Tabela 2.

As formulações apresentaram uma coloração marrom característica dos extratos de açaí e castanha-do-pará, mostrando a influência dos extratos na cor das amostras. A característica perolada provem da base perolizante Surfax. Todas as formulações apresentaram odor agradável, característico da essência de açaí, no entanto muitas não apresentaram um aspecto homogêneo e translúcido.

Tabela 2 - C Resultados dos testes realizados e o aspecto organoléptico das formulações.

\begin{tabular}{|c|c|c|c|}
\hline Formulação & Aspecto & Centrifugação & Cond \pm DP \\
\hline 1 & $\begin{array}{l}\text { Heterogêneo / Translúcido / Muito } \\
\text { Fluido }\end{array}$ & $\begin{array}{l}\text { Presença de } \\
\text { precipitado }\end{array}$ & $4,43 \pm 0,43$ \\
\hline 2 & $\begin{array}{c}\text { Homogêneo / Translúcido / Pouco } \\
\text { Fluido }\end{array}$ & $\begin{array}{l}\text { Presença de } \\
\text { precipitado }\end{array}$ & $7,34 \pm 0,46$ \\
\hline 3 & Homogêneo / Translúcido / Fluido & Homogêneo & $5,75 \pm 0,05$ \\
\hline 4 & $\begin{array}{c}\text { Homogêneo / Translúcido / Pouco } \\
\text { Fluido }\end{array}$ & Homogêneo & $6,82 \pm 0,41$ \\
\hline 5 & Homogêneo / Translúcido / Fluido & Homogêneo & $6,44 \pm 0,27$ \\
\hline 6 & $\begin{array}{c}\text { Heterogêneo / Translúcido / Muito } \\
\text { Fluido }\end{array}$ & $\begin{array}{l}\text { Presença de } \\
\text { precipitado }\end{array}$ & $4,77 \pm 0,03$ \\
\hline 7 & Heterogêneo / Translúcido / Fluido & $\begin{array}{l}\text { Presença de } \\
\text { precipitado }\end{array}$ & $5,16 \pm 0,42$ \\
\hline 8 & $\begin{array}{c}\text { Homogêneo / Translúcido / Pouco } \\
\text { Fluido }\end{array}$ & Homogêneo & $6,99 \pm 0,67$ \\
\hline 0 & Heterogêneo / Translúcido / Fluido & $\begin{array}{l}\text { Presença de } \\
\text { precipitado }\end{array}$ & $6,34 \pm 0,31$ \\
\hline
\end{tabular}


O xampu comercial Nativa SPA apresentou condutividade elétrica de 3,01 $\pm 0,19$ $\mu \mathrm{S} / \mathrm{cm}$ e foi o xampu escolhido para ser o paramêtro de comparação com os xampus elaborados.

As formulações 1, 6 e 7 apresentaram a formação de um precipitado escuro, que pode ser proveniente do excesso de extratos de açaí e de castanha-do-pará, já que a coloração do precipitado é semelhante à do extrato. A formulação 2 apresentou um precipitado com coloração mais clara decorrente do excesso do perolizante surfax utilizado nas formulações.

Condutividade elétrica: Quantifica a capacidade de uma solução de conduzir corrente elétrica e depende do tipo e do número de íons contida na mesma (Machado, 2008).

Analisando o gráfico de Pareto na Figura 1, verifica-se que o componente lauril foi o fator que mais afetou significativamente a condutividade elétrica das amostras.

A alta influência do tensoativo aniônico e a pequena influência do tensoativo catiônico ocorreu porque a condutividade elétrica mede a mobilidade das espécies iônicas em solução e depende do número de íons presentes (Machado, 2008).

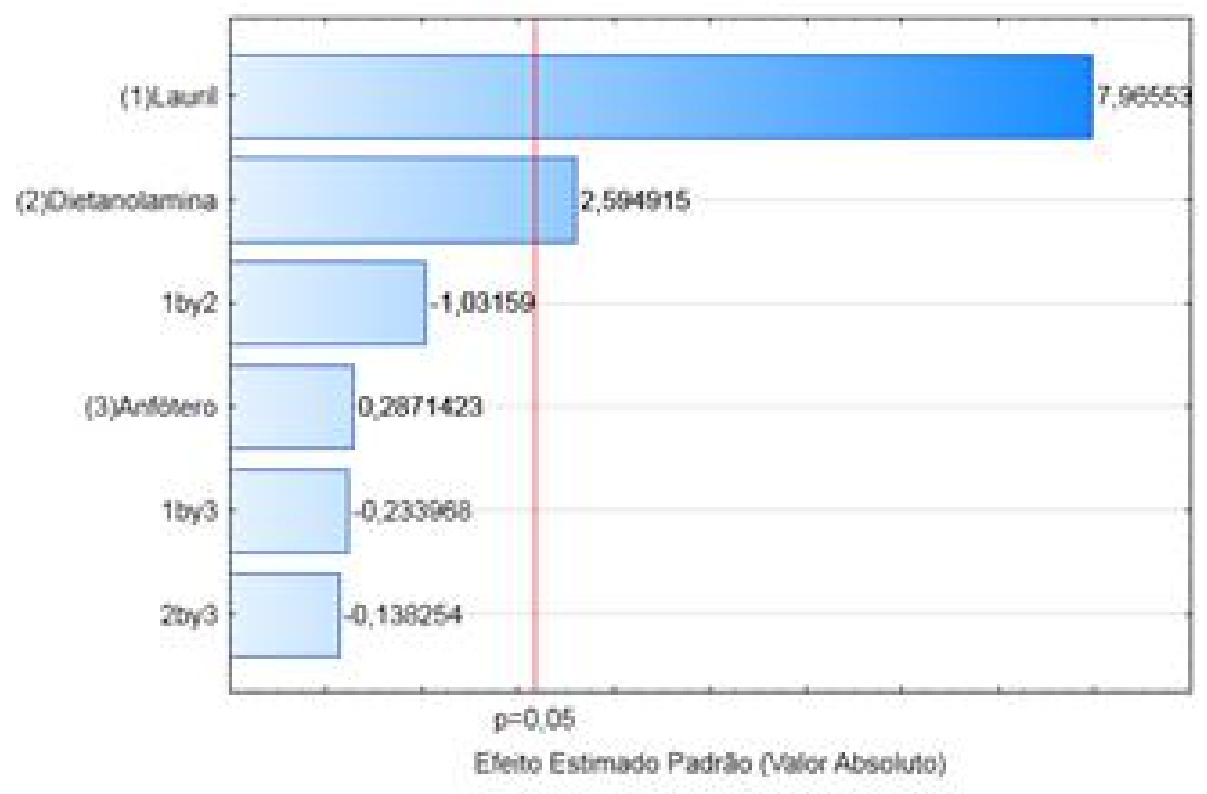

Figura 1- Gráfico de Pareto do planejamento fatorial com ponto central obtido para condutividade (a linha vertical define 5\% de significância estatística).

Verifica-se que a formulações 1 e 6 apresentaram resultados aproximados dos xampus conhecidos. Os valores escolhidos foram os menores valores, porque valores mais altos geram ressecamento e estresse nos cabelos (Jezini, 2013). No entanto, tais formulações formaram precipitado durante a centrifugação, logo devem ser descartadas. Assim, dentre os xampus homogêneos, a formulação 3 , foi a formulação que apresentou o menor valor de condutividade, porém ainda com uma diferença significativa de $91,03 \%$ do xampu comercial com maior condutividade elétrica. 
Dessa forma, percebe-se que é necessário corrigir a condutividade das formulações desenvolvidas diminuindo a quantidade de lauril, o componente que mais influencia nesta propriedade física.

\section{CONCLUSÕES}

As formulações que apresentaram melhores valores de condutividade elétrica foram as formulações 1 e 6, porém foram descartadas devido a formação de precipitado, sendo então o melhor valor de condutividade da formulação 3. Na centrifugação, as formulações 1, 2, 6 e 7 apresentaram formação de precipitado. Com esses resultados, verifica-se a necessidade de ajustar a quantidade de lauril presente nas formulações.

\section{REFERÊNCIAS}

CASTELI, V. C.; MENDONÇA, C. C.; CAMPOS, M. A. L.; FERRARI, M.; MACHADO, S. R. P. Desenvolvimento e estudos de estabilidade preliminares de emulsões O/A contendo Cetoconazol 2,0\%. Revista Maringá, v. 30, n. 2, p. 121-128, 2008.

FARIA, A. B.; PERES, D. D.; VLADI, T. M. K.; CONSIGLIERI, O.; VELASCO, M. V. R.; BABY, A. R. Desenvolvimento e avaliação de produtos cosméticos para a higiene capilar contendo tensoativos "não-sulfatados". Revista de Ciências Farmacêuticas Básica e Aplicada, v. 33, n.4, p. 521 - 527, 2012.

FUJIWARA, G. M.; COSTA, C. K.; ZANIN S. M. W.; MIGUEL, M.D. Avaliação de diversas formulações de xampus de cetoconazol quanto ao emprego de diferentesantioxidantes e solubilizantes. Revista Visão Acadêmica, v. 10, n. 2, p. 43 57, 2009.

GIOKAS, D. L.; SAKKAS, V. A.; ALBANIS, T. A.; LAMPROPOULOU, D. A. Determination of UV-filter residues in bathing waters by liquid chromatography UVdiode array and gas-chromatography-mass spectrometry after micelle mediated extraction-solvent back extraction. Journal of Chromatography A, v. 1077, n. 1, p. 19-27, jun. 2005.

JEZINI, V. S. Uso de planejamento estatístico experimental aplicado ao desenvolvimento de xampu anticaspa com extratos de alecrim e arnica. 2013. Trabalho de conclusão de curso em Engenharia Química. Universidade do Estado do Amazonas. M 2013.

LINS, B. F. E. Ferramentas básicas da qualidade. Ciência da Informação, v. 22, n. 2, p. 153 - 161. 1993.

MACHADO, A. R. J. Avaliação das Características Físico-químicas e Biológicas dos Resíduos de Tanques Sépticos Coletados por Caminhões Limpa-fossas na Cidade de Tubarão-SC. Dissertação (Mestrado em Engenharia Ambiental). Universidade Federal de Santa Catarina. Florianópolis. Santa Catarina. 2008.

PASTORE, F. J., ARAÚJO, V. F., PETRY, A.C., ECHEVERRIA, R. M., FERNANDES, E. C. Plantas da Amazônia para a produção cosmética: uma abordagem química- 60 espécies do extrativismo florestal não madeireiro da Amazônia [Internet]. Brasília: UnB, O I M T e FEPA D ; 2005.

PORTINHO, J. A.; ZIMMERMANN, L. M.; BRUCK, M. R. Efeitos benéficos do açaí. 
International Journal of Nutrology, v.5, n.1, p.15-20, jan./abr., 2012.

ROCHA, C. P. Otimização da Produção de Enzimas por Arpergillus níger em Fermentação em Estado Sólido. Dissertação (Mestrado em Engenharia Química). Faculdade de Engenharia Química. Universidade Federal de Uberlândia. Uberlândia, Minas Gerais, 2010.

SILVA, G. F.; REIS, D. C.; ROCHA, L. A. G.; ALBUQUERQUE, P. M.; CASTILHO, R. B. Avaliação dos parâmetros físico-químicos e organolépticos de xampus elaborados com extratos glicólicos de mulateiro e andiroba. Congresso Brasileiro de Química, 2014.

SOARES, P. H. S, COELHO, R. Z, SILVA, G. F, CASTILHO, R. B, ALBUQUERQUE, P. M. Análise do fator de proteção solar em extratos glicólicos e óleos fixos de plantas da Amazônia. Congresso Brasileiro de Engenharia Química em Iniciação Cientifica. Universidade do Estado do Amazonas, 2015. 